\title{
Quantitative LC-MS/MS uncovers the regulatory role of autophagy in immune thrombocytopenia
}

\author{
Rui-Jie Sun ${ }^{1 \dagger}$, Dong-mei Yin² ${ }^{2 \dagger}$, Dai Yuan ${ }^{1,3}$, Shu-yan Liu1, Jing-jing Zhu ${ }^{3}$ and Ning-ning Shan ${ }^{1,3^{*}}$ (D)
}

\begin{abstract}
Background: Immune thrombocytopenia (ITP) is an autoimmune haemorrhagic disease whose pathogenesis is associated with bone marrow megakaryocyte maturation disorder and destruction of the haematopoietic stem cell microenvironment.

Methods: In this study, we report the qualitative and quantitative profiles of the ITP proteome. Liquid chromatography-tandem mass spectrometry (LC-MS/MS) was conducted to elucidate the protein profiles of clinical bone marrow mononuclear cell (BMMC) samples from ITP patients and healthy donors (controls). Gene Ontology (GO) and Kyoto Encyclopaedia Genes and Genome (KEGG) pathway analyses were performed to annotate the differentially expressed proteins. A protein-protein interaction (PPI) network was constructed with the BLAST online database. Target proteins associated with autophagy were quantitatively identified by parallel reaction monitoring (PRM) analysis.
\end{abstract}

Results: Our approaches showed that the differentially expressed autophagy-related proteins, namely, HSPA8, PARK7, YWHAH, ITGB3 and CSF1R, were changed the most. The protein expression of CSF1R in ITP patients was higher than that in controls, while other autophagy-related proteins were expressed at lower levels in ITP patients than in controls.

Conclusion: Bioinformatics analysis indicated that disruption of the autophagy pathway is a potential pathological mechanism of ITP. These results can provide a new direction for exploring the molecular mechanism of ITP.

Keywords: LC-MS/MS, Immune thrombocytopenia, Autophagy

\section{Introduction}

Immune thrombocytopenia (ITP) is a multifactorial bleeding disease characterized by a breakdown of immune tolerance leading to a platelet count decline. Bone marrow fluid is a vital bodily tissue that has been extensively studied to elucidate the physiology and pathology of the haematological system. Characteristic pathological changes in megakaryocytes suggest

\footnotetext{
*Correspondence: snning@126.com

${ }^{+}$Rui-Jie Sun and Dong-mei Yin contributed equally to this article ${ }^{1}$ Department of Hematology, Shandong Provincial Hospital, Cheeloo College of Medicine, Shandong University, Jinan 250021, Shandong, China

Full list of author information is available at the end of the article
}

an important role of bone marrow in ITP. Blocking the maturation of megakaryocytes $[1,2]$ and variations in the bone marrow microenvironment [3] are important contributors to platelet destruction and/or suppression of platelet production in subjects with ITP [4].

Autophagy, also called self-feeding, is a highly conserved catabolic process in eukaryotic cells. Autophagy is involved in cell development, intracellular quality control, adaptation, starvation, ageing, tumour suppression, innate immunity, and other processes [5]. Autophagy also plays an important role in maintaining the microenvironment and stemness of haematopoietic stem cells [5] and in regulating megakaryopoiesis and platelet function [6]. Wang et al. reported that autophagy was inhibited by bafilomycin A1 or induced by rapamycin in bone marrow 
cells and observed a significant decrease in high-ploidy megakaryocytes and reductions in CD41 and CD61 (two megakaryocytic cell markers) coexpressing cells, proplatelets and platelet formation [7]. Our previous study performed using the human Dami cell line confirmed that autophagy is involved in megakaryocyte endomitosis and platelet development in vitro [8]. As emerging roles of abnormal autophagy in megakaryopoiesis, thrombopoiesis and platelet function have been revealed in ITP patients, insights into signalling pathways may guide future research in this field.

As a powerful technique for biomarker discovery, proteomic strategies have been utilized to study many haematological system malignant diseases, such as acute myelogenous leukaemia (AML) [9] and myelodysplastic syndrome (MDS) [10], revealing the great potential of bone marrow in biomarker discovery and clinical tests. However, bone marrow mononuclear cell (BMMC) protein expression in the context of ITP has not been analysed, which is necessary for deeper pathogenesis research.

In this study, we established an integrated workflow based on the combination of proteome quantification and PRM validation of bone marrow from ITP patients and healthy controls. With the help of advanced bioinformatics, we hoped to further our understanding of the pathogenesis and provide a novel therapeutic approach for ITP. We performed Gene Ontology (GO), Kyoto Encyclopaedia Genes and Genome (KEGG), protein domain enrichment and clustering analyses to determine the correlations between the functions and differential expression of proteins. PRM analysis further confirmed that the expression of autophagy-related proteins (HSPA8, PARK7, YWHAH, ITGB3 and CSF1R) was significantly different in ITP patients and healthy controls. In addition, we indicated that the five autophagy-related differentially expressed proteins were closely related to mammalian target of rapamycin (mTOR) signalling or similar pathways (such as mitogen-activated protein kinase (MAPK) to thereby regulate autophagy activity, and these results may provide useful diagnostic and targeted treatment information for ITP patients in the future.

\section{Materials and methods}

Patient and control samples

The ethics protocol for the collection of human bone marrow aspirate with informed consent was approved by Shandong Provincial Hospital affiliated with Shandong University and Shandong Provincial Hospital affiliated with Shandong First Medical University (ethics approval number: No. 2021-292). Twenty newly diagnosed primary ITP patients in the active phase $(12$ females and 8 males, age range of $18-70$ years, median of 42 years) and 20 healthy controls (12 females and 8 males, age range of $18-55$ years, median of 46 years) were enrolled in this study between May and November 2018. All research participants, including the patients and healthy controls (donors), provided informed consent. The diagnosis of ITP was made according to recently published criteria, including patient history, complete blood count, physical examination and peripheral blood smear examination [11]. The patients' platelet counts ranged from 1 to $30 \times 10^{9} / \mathrm{l}$, with a median platelet count of $11 \times 10^{9} / 1$ (Table 1) [12]. Patients and controls were divided into

Table 1 Clinical characteristics of ITP patients

\begin{tabular}{|c|c|c|c|c|c|}
\hline Group & Group 1 & Group 2 & Group 3 & Group 4 & Median (min.-max.) \\
\hline \multirow[t]{5}{*}{ Age(year)/sex } & $19 / F$ & $18 / F$ & $18 / F$ & $43 / F$ & $42(18-70)$ \\
\hline & $38 / F$ & $27 / M$ & $33 / \mathrm{M}$ & $69 / F$ & \\
\hline & $43 / F$ & $41 / F$ & $48 / F$ & $48 / F$ & \\
\hline & $55 / M$ & $45 / \mathrm{M}$ & $43 / F$ & $25 / M$ & \\
\hline & $70 / \mathrm{M}$ & $65 / F$ & $60 / M$ & $39 / M$ & \\
\hline \multirow[t]{5}{*}{ Platelet counts $\left(\times 10^{9} / \mathrm{l}\right)$} & 9 & 30 & 11 & 1 & $11(1-30)$ \\
\hline & 7 & 18 & 11 & 8 & \\
\hline & 12 & 17 & 12 & 3 & \\
\hline & 3 & 8 & 4 & 12 & \\
\hline & 14 & 29 & 4 & 14 & \\
\hline \multirow[t]{5}{*}{ Bleeding symptoms } & $\mathrm{EC}, \mathrm{PT}$ & EC & PT & PT, GH & \\
\hline & GUH, PT & NONE & $\mathrm{GH}$ & PT, GUH & \\
\hline & $\mathrm{GH}, \mathrm{EP}$ & GUH, PT & $\mathrm{GH}$ & PT, GH & \\
\hline & $\mathrm{GH}$ & $\mathrm{GH}, \mathrm{EC}$ & $\mathrm{EP}, \mathrm{GH}$ & $\mathrm{GH}, \mathrm{EP}$ & \\
\hline & NONE & $\mathrm{EP}, \mathrm{GH}$ & $\mathrm{EC}, \mathrm{GH}$ & $\mathrm{GH}$ & \\
\hline
\end{tabular}


four groups according to their age and sex to reduce differences and increase the accuracy of the results. None of the subjects had been treated with glucocorticosteroids, immunoglobulins or immunosuppressants prior to sampling. Bone marrow aspirate was collected into heparin-containing vacutainer tubes. According to the manufacturer's instructions, BMMCs were isolated from heparinized bone marrow aspirate samples by FicollPaque gradient centrifugation (Pharmacia Diagnostics, Uppsala, Sweden). BMMCs from ITP and control subjects were stored at $-80^{\circ} \mathrm{C}$.

\section{Crude protein extraction and trypsin digestion}

The samples were centrifuged at $12,000 \mathrm{~g}$ for $10 \mathrm{~min}$ at $4{ }^{\circ} \mathrm{C}$, and the cell debris was discarded. After transferring the supernatant to a new centrifuge tube, the protein was precipitated with $20 \%$ cold trichloroacetic acid (TCA) and washed with cold acetone. An Abundant Protein Depletion Kit (Pierce Top 12, Thermo) was used to remove the highly abundant proteins. The proteins were redissolved in buffer $(8 \mathrm{M}$ urea, $100 \mathrm{mM}$ TEAB, $\mathrm{pH}$ 8.0), and the protein concentration was determined with a BCA kit. The protein solution was digested with $5 \mathrm{mM}$ dithiothreitol (Sigma) at $56^{\circ} \mathrm{C}$ for $30 \mathrm{~min}$ and then alkylated with $11 \mathrm{mM}$ iodoacetamide (Sigma) in the dark at room temperature for $15 \mathrm{~min}$. The protein sample was then diluted with $100 \mathrm{mM}$ TEAB to decrease the urea concentration to less than $2 \mathrm{M}$. Finally, trypsin was added at a 1:50 trypsin-to-protein mass ratio for the first digestion overnight and at a 1:100 trypsin-to-protein mass ratio for a second $4 \mathrm{~h}$ digestion to improve the digestion effect $[13,14]$.

\section{High-performance liquid chromatography (HPLC) fractionation and LC MS/MS analysis}

Tryptic peptides were fractionated by high $\mathrm{pH}$ reversephase HPLC on an Agilent 300Extend C18 column ( $5 \mu \mathrm{m}$ particles, $4.6 \mathrm{~mm}$ ID, $250 \mathrm{~mm}$ length). Peptides were separated into 60 fractions with an acetonitrile ( $\mathrm{pH} 9.0)$ gradient of $8-32 \%$ over $60 \mathrm{~min}$. They were then combined into 4 fractions and dried by vacuum centrifugation. The peptides were redissolved in solvent A $(0.1 \%$ formic acid in $2 \%$ acetonitrile) and loaded onto a reverse-phase analytical pre-column (Acclaim PepMap 100, Thermo Scientific). The gradient was as follows: $6-25 \%$ solvent B $(0.1 \%$ formic acid in $90 \%$ acetonitrile) over $40 \mathrm{~min}$; $25-35 \%$ over $12 \mathrm{~min}$; $35-80 \%$ over $4 \mathrm{~min}$; and holding at $80 \%$ for $4 \mathrm{~min}$. An EASY-nLC 1000 UPLC system was utilized at a constant flow rate of $500 \mathrm{~nL} / \mathrm{min}$. The peptides were subjected to a nanospray ionization (NSI) source on the Q Exactive ${ }^{\mathrm{TM}}$ Plus (Thermo) instrument coupled online to the UPLC and detected by the Orbitrap. A data-dependent procedure (DDA) that alternated between one MS scan followed by 20 tandem mass spectrometry (MS/MS) scans was performed. Automatic gain control (AGC) was used to prevent overfilling of the Orbitrap, and 5E4 ions were accumulated for the generation of MS/MS spectra; the maximum injection time was set at $30 \mathrm{~ms}$, and the signal threshold was set at 15,000 ions/s.

\section{Bioinformatics/functional enrichment analyses}

The resulting MS/MS data were processed using the Maxquent search engine (v.1.5.2.8).

InterProScan software was used to identify protein domain functions based on the protein sequence alignment method. Then, proteins were classified by GO annotation based on three categories: biological process, cellular component and molecular function. The KEGG online service tool KAAS was used to annotate the protein KEGG database descriptions. The annotation results were then mapped to the KEGG pathway database using KEGG mapper. The GO and KEGG analysis results were subjected to two-tailed Fisher's exact tests to determine the enrichment of the differentially expressed proteins against all identified proteins. A corrected p-value $<0.05$ was considered significant. All categories, and their $\mathrm{p}$ values, were collated after enrichment, and those that were enriched in at least one of the clusters and had a $\mathrm{p}$ value $<0.05$ were filtered. The clusters were visualized as a heat map using the "heatmap.2" function. All differentially expressed proteins were searched against the STRING database version 10.1 for protein-protein interactions (PPIs) and visualized with the $\mathrm{R}$ package "networkD3".

\section{Parallel reaction monitoring (PRM) analysis}

Target proteins associated with autophagy were quantitatively identified using mass spectrometry-based targeted proteome quantification. The proteins selected for PRM were based on the quantitative analysis of the bone marrow serum proteome. The crude protein extraction and trypsin digestion steps were similar to those described above. The electrospray voltage was $2.0 \mathrm{kV}$, and the peptide length was set to $7-25$. The transition settings were as follows: precursor charges were set to 2 and 3, ion charges were set to 1 , and ion types were set to $b$ and $y$. The product ions were set from ion 3 to the last ion, and the ion match tolerance was set to $0.02 \mathrm{Da}$. The PRM data were analysed using Skyline (v.3.6) software.

\section{Statistical analysis}

Statistical analyses were performed using GraphPad Prism 8 software. For PRM data analysis, two groups were compared by two-tailed Student's t-tests. A p-value $<0.05$ was considered statistically significant. 


\section{Results}

\section{Proteome quantification overview}

In this study, we established an integrated method for quantifying the proteome of human BMMCs. We assessed the data accuracy and validated the sample preparations meeting the requirements; we identified 829 proteins in human BMMCs, among which 613 proteins were quantified. In total, 69 proteins were downregulated with a fold change $<1 / 1.5$, while 26 proteins were upregulated with a fold change $>1.5(\mathrm{p} \leq 0.05)$. To identify differentially expressed proteins, volcano plot analysis was conducted to visualize the differences between the ITP patient and control groups (Fig. 1).

\section{Abnormal regulation of autophagy-related proteins (HSPA8, PARK7, YWHAH, ITGB3, and CSF1R)}

To identify the functional classifications and pathways of the differentially expressed proteins in ITP, we performed KEGG pathway enrichment analysis. Our data showed that the upregulated differentially expressed proteins in ITP were most prominently enriched in the complement and coagulation cascades and that the downregulated differentially expressed proteins in ITP were enriched in the carbon metabolism (Fig. 2A), regulation of actin cytoskeleton, vasopressin-regulated water reabsorption and tight junction pathways (Fig. 2B). Using the networkD3 $\mathrm{R}$ package, we revealed some highly connected subnetworks among autophagy proteins, including heat shock

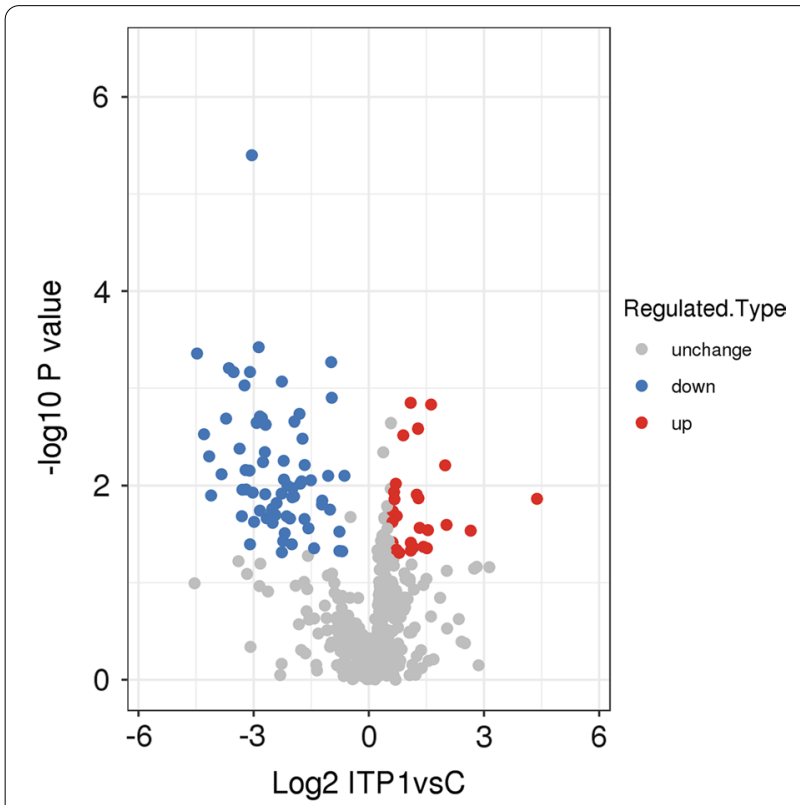

Fig. 1 Volcano plots of all proteins identified by LC-MS/MS analysis. The red dots in these plots represent the upregulated proteins with statistical significance, and the blue dots represent the downregulated proteins (fold change $\geq 1.5, p \leq 0.05$ ) protein family A (Hsp70) member 8 (HSPA8), Parkinson's disease (autosomal recessive, early onset) 7 (PARK7), tyrosine 3-monooxygenase/tryptophan 5-monooxygenase activation protein, eta polypeptide, isoform CRA_b (YWHAH), integrin beta-3 (ITGB3) and colony-stimulating factor 1 receptor (CSF1R). The expression of the CSF1R protein in ITP patients was higher than that in controls, while the other autophagy-related proteins were expressed at lower levels in ITP patients than in controls.

The downregulated protein HSPA8 participates in signalling pathways associated with the longevity of multiple species and endocytosis (Additional file 1: Table S1). YWHAH was downregulated and enriched in the cell cycle, vasopressin-regulated water reabsorption and Hippo signalling pathways, and ITGB3 was downregulated and enriched in the focal adhesion, platelet activation and haematopoietic cell lineage pathways (Additional file 1: Table S1). The autophagy-related CSF1R protein was upregulated and closely related to the haematopoietic cell lineage and cytokine-cytokine receptor interactions (Additional file 1: Table S1).

Protein functions are largely dependent on specific domain structures in their sequence. To assess the domain structures, a bubble chart was constructed by Fisher's exact test $[\log 2(\mathrm{p}$ value $)]$ to analyse protein domain enrichment. In agreement with our findings, the protein domains associated with lipocalin/cytosolic fattyacid binding and calycin were enriched in the upregulated proteins (Fig. 2C) in ITP, while the thioredoxin-like fold was the most enriched domain among the downregulated proteins (Fig. 2D). CSF1R, an autophagy-related protein, was mainly related to the immunoglobulinlike domain, serine-threonine/tyrosine-protein kinase catalytic domain and tyrosine-protein kinase catalytic domain (Additional file 2: Table S2). Some important domains of other downregulated autophagy proteins were enriched, such as the 14-3-3 domain of YWHAH and the C-terminal domain of HSPA8 (Additional file 2: Table S2).

\section{Clustering analysis}

To assess the correlations between the functions of differentially expressed proteins in the ITP patient group, all the differentially expressed proteins were divided into four quantiles (Q1-Q4) according to their expression ratios as described above. Then, enrichment-based clustering analyses (GO, KEGG and protein domain) were performed (Fig. 3).

The biological process category was analysed as shown in Fig. 3A. The downregulated proteins were highly enriched in the platelet activation, cell-cell adhesion, and integrin-mediated signalling (Q1) terms, which may be associated with the autophagy process. 


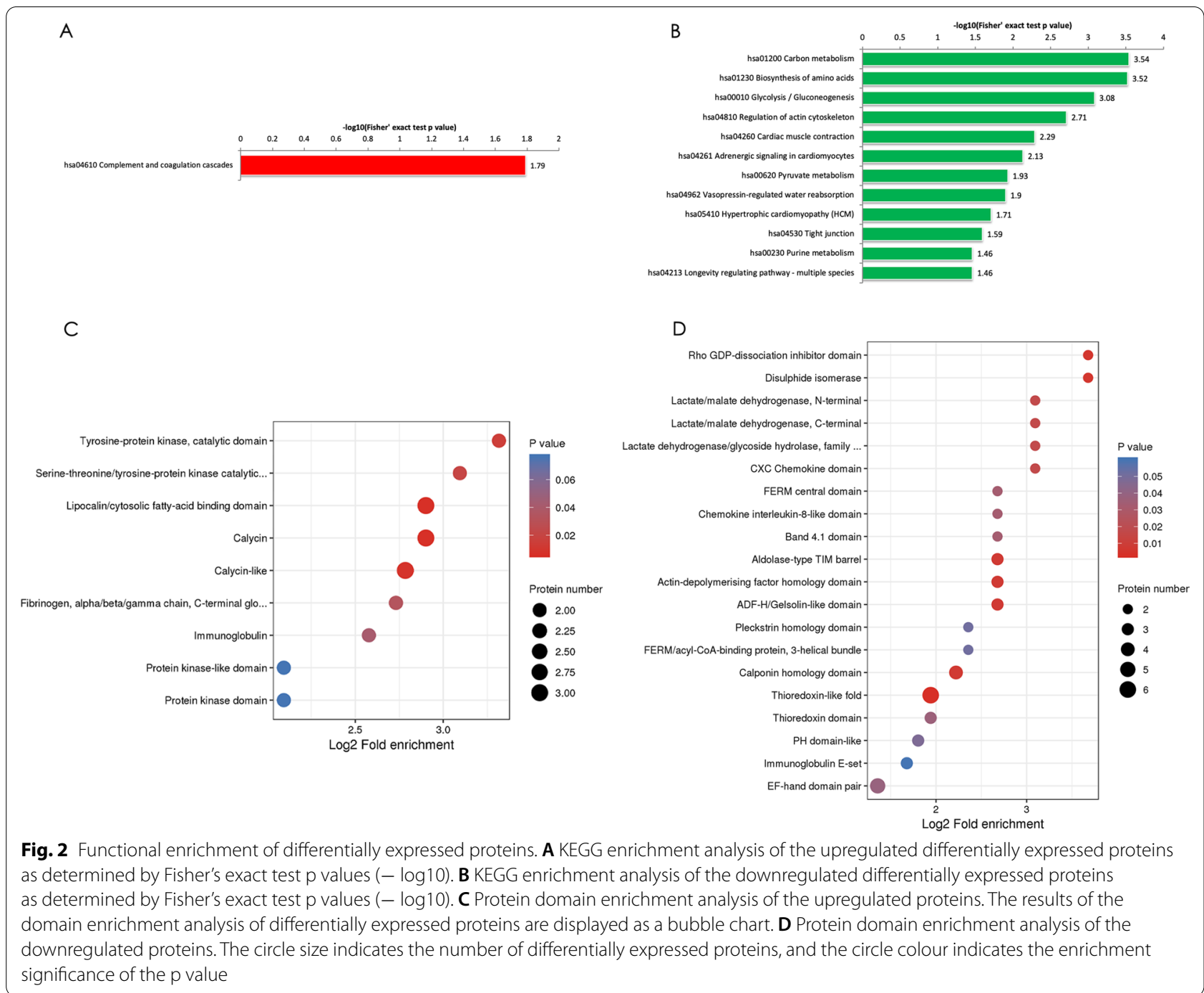

The upregulated proteins were highly enriched in the negative regulation of adaptive immune response term in Q3 and in the acute inflammatory response, positive regulation of adaptive immune response and negative regulation of cytokine production terms in Q4, which may be attributed to the mechanism underlying the unbalanced autoimmune response in ITP. In the cellular component category, the upregulated proteins were mainly located in the organism cell part (Q3) and in the extracellular space and fibrinogen complex (Q4), while the downregulated proteins were mainly located in the cytoplasmic region (Fig. 3B). The results of molecular function analysis are presented in Fig. 3C. Downregulated proteins with small molecule binding activity, signalling adaptor activity and transcription coactivation activity were enriched in Q1. The chaperone binding function of upregulated proteins was enriched in Q4.
KEGG pathway analysis of the differentially expressed proteins in ITP revealed several vital pathways (Fig. 4D). The tight junction pathway was enriched in Q1. The upregulated proteins were associated with the platelet activation pathway in Q4. CSF1R, YWHAH and ITGB3 were enriched in the PI3K/Akt/mTOR signalling pathway (Additional file 3: Table S3). In addition, CSF1R and HSPA8 were shown to participate in the MAPK signalling pathway, which plays crucial roles in various antiproliferative events, including apoptosis and autophagy [15]. Therefore, autophagy-related proteins (HSPA8, CSF1R, YWHAH and ITGB3) were shown to be involved in the pathogenesis of ITP by affecting autophagy and its related pathways.

Domain enrichment analysis of differentially expressed proteins (Fig. 3E) showed that the downregulated proteins clustered with the immunoglobulin E-set, thioredoxin domain and chemokine interleukin-8-like domain, 


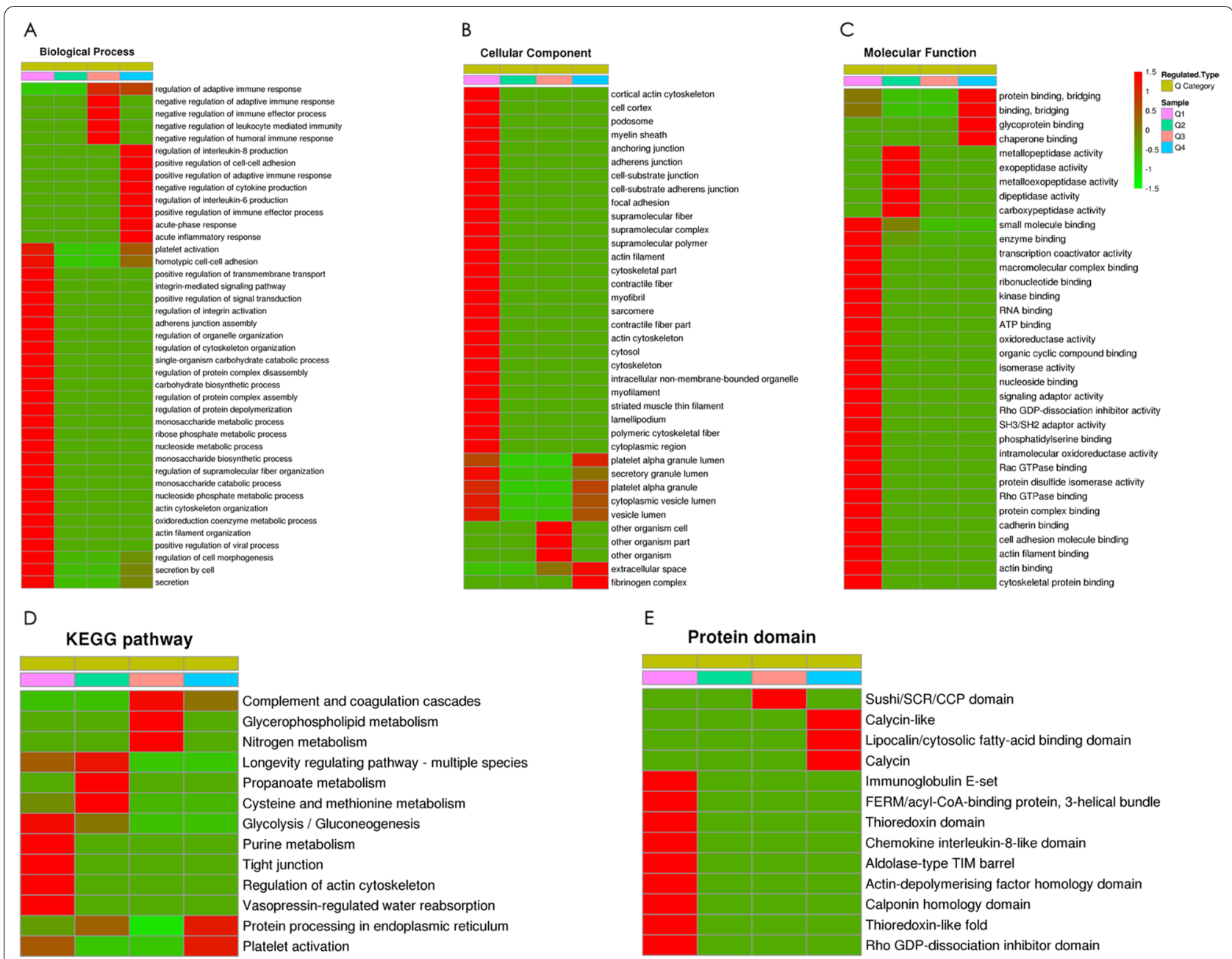

Fig. 3 Heatmaps of clusters based on the enrichment of (A) GO biological processes, (B) GO cellular components, (C) GO molecular functions, (D) KEGG pathways, and (E) protein domains. The differentially expressed proteins were divided into four quantitative categories according to the ITP patient group (P) to control group (C) ratio: $\mathrm{Q} 1(0<\mathrm{P} / \mathrm{C}$ ratio $<1 / 2$, $\mathrm{P}$ value $<0.05)$ and $\mathrm{Q} 2(1 / 2<\mathrm{P} / \mathrm{C}$ ratio $<1 / 1.5$, $\mathrm{P}$ value $<0.05)$ represent downregulated proteins, and $\mathrm{Q} 3(1.5<\mathrm{P} / \mathrm{C}$ ratio $<2$, $\mathrm{p}$ value $<0.05)$ and $\mathrm{Q} 4(\mathrm{P} / \mathrm{C}>2$, $\mathrm{p}$ value $<0.05)$ represent upregulated proteins

while the upregulated proteins clustered with the fibrinogen, alpha/beta/gamma chain and calycin-like lipocalin/ cytosolic fatty-acid binding domains.

\section{PPI network}

We visualized a PPI network of all quantified proteins using the Search Tool for the Retrieval of Interacting Genes/Proteins (STRING) (V.10.5) database. A complete network of differentially expressed proteins was created and included 50 interactions. We found that autophagyrelated proteins were clearly upregulated (CSF1R) in the ITP patient group, while a number of proteins (HSPA8, PARK7, YWHAH, and ITGB3) related to autophagy were downregulated. Our data set offers insights into the probability of interactions among autophagy proteins in ITP. A representative example is shown in Fig. 4A.

\section{PRM analysis}

PRM quantification was carried out for 20 selected target proteins in all samples. Limited by the characteristics of some proteins and the abundance of their expression, we quantified 14 of these selected target proteins. The peak area was used for PRM quantitative analysis. The PRM quantitative results for autophagy-related proteins, shown in Fig. 4B, further verified that HSPA8, PARK7, YWHAH, and ITGB3 were downregulated and that CSF1R was upregulated in the ITP patient group compared with the control group. 


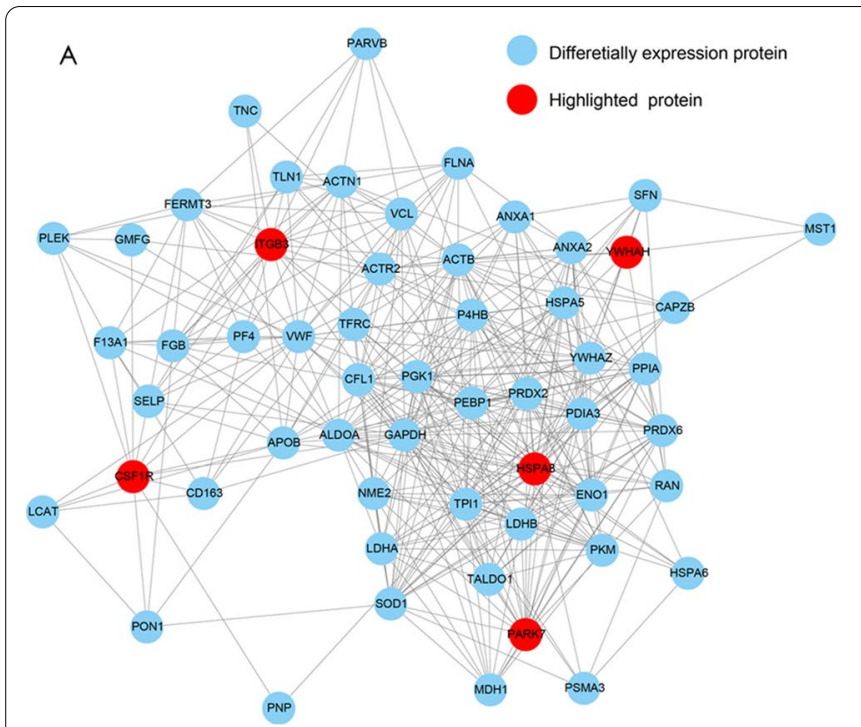

B

Fig. 4 PPI network and PRM analysis of autophagy-related proteins. A A complete PPI network was constructed. Five autophagy-related proteins (HSPA8, PARK7, YWHAH, ITGB3 and CSF1R) were highlighted among the differentially expressed proteins. B The quantitative analysis results of autophagy-related proteins (HSPA8, PARK7, YWHAH, ITGB3 and CSF1R) were verified by PRM. With the exception of CSF1R, these autophagy-related proteins was significantly downregulated in the ITP group compared with the control group. The data are presented as the mean \pm SD; two-tailed Student's t-test, ${ }^{*} p<0.05,{ }^{* *} p<0.01,{ }^{* * *} p<0.001$

\section{Discussion}

Proteomics has the potential to provide clues to cancer and autoimmune disease pathogeneses based on comprehensive analyses of protein expression and activation statuses. In our study, we employed a quantitative proteomics strategy to compare the autophagy-related proteins that were differentially expressed in the BMMCs of ITP patients and control subjects and to explore the potential mechanism of ITP pathogenesis. The data showed that 26 upregulated proteins in ITP were enriched in the acute-phase response and regulation of the adaptive immune response, while 69 downregulated proteins were enriched in processes associated with binding, such as actin binding and cytoskeletal protein binding, which manifested as changes in the autophagy fractions. Then, we detected 5 abnormally expressed autophagy-related proteins that may be associated with the pathogenesis of ITP, among which 4 (HSPA8, PARK7, YWHAH, and ITGB3) were downregulated and 1 (CSF1R) was significantly upregulated in ITP patients compared with the controls. Clustering analysis showed that most of the autophagy-related differentially expressed proteins (YWHAH, ITGB3 and CSF1R) identified herein were closely related to the PI3K/Akt/ mTOR signalling pathway. The mTOR signalling pathway mediates many physiological functions, such as cell proliferation, differentiation, migration and apoptosis, and regulates autophagy $[16,17]$. Studies have shown that the pathway controlling mTOR expression negatively regulates autophagy in cells stimulated by factors such as starvation and hypoxia [18].

YWHAH is a large family of phosphoregulatory proteins that exist primarily as homo- and heterodimers [19]. YWHAH proteins are involved in different signalling pathways that modulate cellular and whole-body energy as well as nutrient homeostasis, such as the insulin and mTOR- and AMP-dependent kinase (AMPK) signalling pathways, and regulate autophagy [20]. Considerable cross-talk exists between the AMPK pathway and other key energy regulatory pathways, such as insulin signalling and mTOR signalling complex 1 (mTORC1) [20]. AMPK is reported to inhibit mTORC1 by activating tuberous sclerosis proteins 1 and 2 (TSC1/2) and by inhibiting regulatory-associated protein of TOR (RAPTOR) via the phosphorylation-induced binding of YWHAH [21], both of which stimulate autophagy. Recently, a direct stimulatory pathway correlating AMPK with autophagy was described based on the phosphorylation of ULK1 [22], and complex formation among ULK1, mTORC1 and AMPK was found to coincide with the phosphorylation of RAPTOR and the binding of YWHAH [23]. We speculated that abnormal autophagy is associated with ITP in patients with low YWHAH protein expression, as it inhibits RAPTOR, ultimately decreasing the function and quantity of megakaryocytes and platelets and leading to the onset of ITP.

KEGG enrichment analysis of the differentially expressed proteins showed that the downregulated 
autophagy-related protein ITGB3 was also enriched in platelet activation and haematopoietic cell lineages. ITGB3 is an important molecule involved in cell survival, proliferation and cancer metastasis [24]. ITGB3 has been reported to be upstream of the PI3K/AKT/mTOR signalling pathway in various cell types, and the pathway is activated when ITGB3 is overexpressed [25]. Studies have shown that ITGB3 upregulation inhibits the autophagic process in cardiomyocytes by activating $\mathrm{AKT}$, suggesting that the expression status of ITGB3 affects cell autophagy [26]. ITGB3 encodes GP3A (also known as GPIIIa) and represents a common platelet antigen polymorphism (PIA1/A2) that can influence platelet activation and aggregation. ITGB3 can provide instructions for forming the $\beta 3$ subunit of the receptor integrin $\alpha \operatorname{IIb} \beta 3$. ITGB3 mutations cause activation of alphaIIb/beta3 ( $\alpha \operatorname{IIb} \beta 3$ ) and leads to platelet dysfunction and macrothrombocytopenia [27]. Another study found that eltrombopag can induced relevant changes in the hematopoiesis, platelet activation as well as megakaryocyte differentiation with the overexpression of the genes ITGB3 [28].Our results showed that the expression of ITGB3 in the ITP patient group was lower than that in the control group, suggesting that the overexpression of autophagy may be caused by the downregulation of AKT activation by ITGB3.

CSF1R was herein identified as another important autophagy-related protein. Autophagy mediated by CSF-1/CSF1R plays a crucial role during the differentiation of human monocytes into macrophages [29, 30], which induce typical autophagic structures, such as phagophores and autophagosomes, and results in the accumulation of LC3-II [29]. Tian et al. showed that the level of LC3-II was lower in cells overexpressing CSF1R-Mut than in benign control or CSF1R-WT cells exposed to CSF-1 stimulation, indicating that the autophagy process might be disturbed by abnormal CSF-1/CSF1R signalling [31]. At the molecular level, E5[N-(3-((4)(benzofuran-2-yl) pyrimidin-2-yl) oxy)-4-methylphenyl)-4-((4-methylpiperazin-1-yl) methyl) benzamide] was able to downregulate the mTOR pathway and activate the MAPK/ERK pathway [15, 32], thus inducing the conversion of LC3-I to LC3-II, increasing the expression of Atg5 and restoring autophagy. In our study, the expression level of CSF1R was decreased in the control group compared with the ITP patient group, which may demonstrate that abnormal autophagy mediated by CSF1/CSF1R signalling is involved in the pathogenesis of ITP.

HSPA8 was enriched in the longevity regulating pathway-multiple species as determined by KEGG analysis. HSPA8 is a molecular chaperone involved in a wide variety of cellular processes that localizes in the nucleus, cytosol, extracellular exosomes, and cell membrane [33]. HSPA8 detects substrates that are processed by chaperone-mediated autophagy (CMA) [34], and its expression is altered in a number of immune disorders. For example, flow cytometry studies showed that the expression of HSPA8 was increased in the splenic B and T cells of MRL/MpTn-gld/gld lupus-prone mice [35, 36]. HSPA8 was also shown to be involved in the molecular regulation of haematopoiesis [37]. PARK7 is a multifunctional protein that is involved in various cellular activities, and its principle functions include antioxidative defence and mitochondrial homeostasis maintenance [38]. PARK7 dysfunction leads to mitochondrial defects. Furthermore, CMA protects cells from mitochondrial toxin MPPC-induced changes in mitochondrial morphology and function and increases cell viability [39]. Under PARK7-deficient conditions in subjects with ITP, these protective effects may be lost.

In recent years, experimental and clinical evidence has concluded that autophagy plays an important role in maintaining the stemness and microenvironment of haematopoietic stem cells [5]. Perturbations of normal autophagy processes in ITP patients may be caused by the deletion of autophagy-related genes such as ATG7 and abnormal signalling due to the overexpression of mTOR. These changes are thought to affect markers of haematopoietic stem cells, such as CD41 and CD61, and the differentiation of megakaryocytes, ultimately decreasing the function and quantity of platelets and leading to the onset of ITP [3]. Ouseph et al. demonstrated that the autophagy process is essential for the normal functioning of platelet activation and aggregation [40]. In another study, they demonstrated that starvation induced substantial autophagy (above basal level), which was characterized by decreased platelet aggregation, reduced calcium mobilization and granule secretion, decreased adhesion to immobilized fibrinogen, and eventually, an increased bleeding time [41].

In this study, we indicated five autophagy-related differentially expressed proteins in ITP BMMC samples. GO, KEGG, protein domain enrichment and clustering analyses were performed to determine the correlations between the functions and differential expression of proteins. PRM analysis further confirmed that the expression of autophagy-related proteins was significantly different in ITP patients and health controls. CSF1R in ITP patients was higher than that in controls, while other autophagy-related proteins, HSPA8, PARK7, YWHAH, ITGB3, were expressed at lower levels in ITP patients than in controls. Furthermore, we indicated that the five autophagy-expressed proteins were closely related to mTOR signaling or similar pathways that regulate autophagy activity, and these results may provide more strategies for ITP targeted treatments and diagnosis in the future. 
In the follow-up experiments, Western Blot, immunohistochemistry, RT-PCR and other biological techniques will be used to verify the expression levels of five autophagy-related proteins in more specimens. The differential condition of these autophagy-related proteins in the levels of proteins and transcription will be observed in vitro experiments with bafilomycin A1 and rapamycin. The expression level of autophagy differential proteins can be detected after the treatment of in ITP mouse by blocking or enhancing targeted proteins. These will be helpful for further confirmation the relationship between the five autophagy-related proteins (especially YWHAH, CSF1R and ITGB3) and ITP bone marrow megakaryocyte cells and their specific role in PI3K/Akt/mTOR pathway.

\section{Supplementary Information}

The online version contains supplementary material available at https://doi. org/10.1186/s12935-021-02249-4.

Additional file 1: Table 1. The description of KEGG pathway.

Additional file 2: Table 2 . The description of protein domains.

Additional file 3: Table 3. The description of clustering analysis.

\section{Acknowledgements}

Not applicable.

\section{Authors' contributions}

SRJ contributed to the data analysis and wrote the manuscript. YDM contributed to the data analysis. YD, LSY and ZJJ contributed to the statistical analysis. SNN obtained the funding and designed the experiments. All authors read and approved the final manuscript.

\section{Funding}

The work was supported by grants from the Taishan Youth Scholar Foundation of Shandong Province (tsqn201812140), the Academic Promotion Program of Shandong First Medical University (2019RC018), the Taishan Scholar Foundation of Shandong Province, the National Natural Science Foundation of China (81570104), Natural Science Foundation of Shandong Province (ZR202102200578;ZR2020MH112), the Key Research and Development Project of Jinan $(201907021 ; 201907026)$, the Key Research and Development Program of Shandong Province (2018CXGC1213), and the Technology Development Projects of Shandong Province (2017GSF18189).Projects of medical and health technology development program of Shandong province (2019WS498).

\section{Availability of data and materials}

All of the data in this manuscript are presented in the main paper.

\section{Declarations}

\section{Ethical approval and consent to participate}

Informed consent was obtained from each participating patient and/or legal guardian. Ethical approval for the study was obtained from the Medical Ethics Committee of Shandong Provincial Hospital affiliated with Shandong University and Shandong Provincial Hospital affiliated with Shandong First Medical University.

\section{Consent for publication}

Not applicable.

\section{Competing interests}

The authors have no competing interests to declare.

\section{Author details}

${ }^{1}$ Department of Hematology, Shandong Provincial Hospital, Cheeloo College of Medicine, Shandong University, Jinan 250021, Shandong, China. ${ }^{2}$ Department of Blood Transfusion, Shandong Provincial Hospital, Cheeloo College of Medicine, Shandong University, Jinan 250021, Shandong, China. ${ }^{3}$ Department of Hematology, Shandong Provincial Hospital Affiliated to Shandong First Medical University, 324 Jing Wu Rd, Jinan 250021, Shandong, China.

Received: 8 November 2020 Accepted: 7 October 2021

Published online: 18 October 2021

\section{References}

1. Chow $L$, et al. A murine model of severe immune thrombocytopenia is induced by antibody- and CD8 $+T$ cell-mediated responses that are differentially sensitive to therapy. Blood. 2010;115(6):1247-53.

2. Semple JW, et al. Differences in serum cytokine levels in acute and chronic autoimmune thrombocytopenic purpura: relationship to platelet phenotype and antiplatelet T-cell reactivity. Blood. 1996;87(10):4245-54.

3. Sun RJ, Shan NN. Megakaryocytic dysfunction in immune thrombocytopenia is linked to autophagy. Cancer Cell Int. 2019;19:59.

4. Shan NN, et al. Targeting autophagy as a potential therapeutic approach for immune thrombocytopenia therapy. Crit Rev Oncol Hematol. 2016:100:11-5.

5. Matsuura A, et al. Apg1p, a novel protein kinase required for the autophagic process in Saccharomyces cerevisiae. Gene. 1997:192(2):245-50

6. Cao Y, et al. Loss of autophagy leads to failure in megakaryopoiesis, megakaryocyte differentiation, and thrombopoiesis in mice. Exp Hematol. 2015;43(6):488-94.

7. Wang $\mathrm{Q}$, et al. Rapamycin and bafilomycin $\mathrm{A} 1$ alter autophagy and megakaryopoiesis. Platelets. 2017;28(1):82-9.

8. Sun RJ, et al. Rapamycin induces megakaryocytic differentiation through increasing autophagy in Dami cells. Blood Coagul Fibrinolysis. 2020:31(5):310-6.

9. Tibes $R$, et al. Reverse phase protein array: validation of a novel proteomic technology and utility for analysis of primary leukemia specimens and hematopoietic stem cells. Mol Cancer Ther. 2006;5(10):2512-21.

10. Braoudaki $\mathrm{M}$, et al. Proteomic analysis of childhood de novo acute myeloid leukemia and myelodysplastic syndrome/AML: correlation to molecular and cytogenetic analyses. Amino Acids. 2011;40(3):943-51.

11. Provan $D$, et al. International consensus report on the investigation and management of primary immune thrombocytopenia. Blood. 2010;115(2):168-86.

12. Liu SY, et al. Significant reductions in apoptosis-related proteins (HSPA6, HSPA8, ITGB3, YWHAH, and PRDX6) are involved in immune thrombocytopenia. J Thromb Thrombolysis. 2020;51:905.

13. Hahne $\mathrm{H}$, et al. Proteome wide purification and identification of O-GlcNAc-modified proteins using click chemistry and mass spectrometry. J Proteome Res. 2013;12(2):927-36.

14. Helm $D$, et al. Ion mobility tandem mass spectrometry enhances performance of bottom-up proteomics. Mol Cell Proteomics. 2014:13(12):3709-15.

15. Oseini AM, Roberts LR. PDGFRalpha: a new therapeutic target in the treatment of hepatocellular carcinoma? Expert Opin Ther Targets. 2009;13(4):443-54.

16. Chen N, Debnath J. IkappaB kinase complex (IKK) triggers detachmentinduced autophagy in mammary epithelial cells independently of the PI3K-AKT-MTORC1 pathway. Autophagy. 2013;9(8):1214-27.

17. Chang F, et al. Involvement of PI3K/Akt pathway in cell cycle progression, apoptosis, and neoplastic transformation: a target for cancer chemotherapy. Leukemia. 2003;17(3):590-603.

18. Lamoureux F, Zoubeidi A. Dual inhibition of autophagy and the AKT pathway in prostate cancer. Autophagy. 2013;9(7):1119-20.

19. Patrick DM, et al. Defective erythroid differentiation in miR-451 mutant mice mediated by 14-3-3zeta. Genes Dev. 2010;24(15):1614-9. 
20. Kleppe $\mathrm{R}$, et al. The 14-3-3 proteins in regulation of cellular metabolism. Semin Cell Dev Biol. 2011;22(7):713-9.

21. Gwinn DM, et al. AMPK phosphorylation of raptor mediates a metabolic checkpoint. Mol Cell. 2008;30(2):214-26.

22. Inoki K, Kim J, Guan KL. AMPK and mTOR in cellular energy homeostasis and drug targets. Annu Rev Pharmacol Toxicol. 2012;52:381-400.

23. Lee JW, et al. The association of AMPK with ULK1 regulates autophagy. PLOS ONE. 2010;5(11):e15394.

24. Zhou Y, et al. Thy-1-integrin alphav beta5 interactions inhibit lung fibroblast contraction-induced latent transforming growth factorbeta1 activation and myofibroblast differentiation. J Biol Chem. 2010;285(29):22382-93.

25. Wan $\mathrm{H}$, et al. Thy-1 depletion and integrin beta 3 upregulation-mediated PI3K-Akt-mTOR pathway activation inhibits lung fibroblast autophagy in lipopolysaccharide-induced pulmonary fibrosis. Lab Invest. 2019;99(11):1636-49.

26. Zhu Y, et al. ss3-integrin inhibits lipopolysaccharide-induced autophagy in cardiomyocytes via the Akt signaling pathway. Cardiology. 2015;130(4):249-59.

27. Bury $L$, et al. Cytoskeletal perturbation leads to platelet dysfunction and thrombocytopenia in variant forms of Glanzmann thrombasthenia. Haematologica. 2016;101(1):46-56.

28. Hernandez-Sanchez JM, et al. Transcriptomic analysis of patients with immune thrombocytopenia treated with eltrombopag. Platelets. 2020;31(8):993-1000.

29. Jacquel A, et al. Autophagy is required for CSF-1-induced macrophagic differentiation and acquisition of phagocytic functions. Blood. 2012;119(19):4527-31.

30. Li MM, et al. Standards and guidelines for the interpretation and reporting of sequence variants in cancer: a joint consensus recommendation of the association for molecular pathology, American Society of Clinical Oncology, and College of American Pathologists. J Mol Diagn. 2017;19(1):4-23

31. Tian WT, et al. Clinicopathologic characterization and abnormal autophagy of CSF1R-related leukoencephalopathy. Transl Neurodegener. 2019;8:32
32. Zhou Q, Lui VW, Yeo W. Targeting the PI3K/Akt/mTOR pathway in hepatocellular carcinoma. Future Oncol. 2011;7(10):1149-67.

33. Liu T, Daniels CK, Cao S. Comprehensive review on the HSC70 functions, interactions with related molecules and involvement in clinical diseases and therapeutic potential. Pharmacol Ther. 2012;136(3):354-74.

34. Bonam SR, Ruff M, Muller S. HSPA8/HSC70 in immune disorders: a molecular rheostat that adjusts chaperone-mediated autophagy substrates. Cells. 2019;8(8):849.

35. Page N, et al. HSC70 blockade by the therapeutic peptide P140 affects autophagic processes and endogenous $\mathrm{MHCll}$ presentation in murine lupus. Ann Rheum Dis. 2011;70(5):837-43.

36. Wang $F$, Muller $S$. Manipulating autophagic processes in autoimmune diseases: a special focus on modulating chaperone-mediated autophagy, an emerging therapeutic target. Front Immunol. 2015;6:252.

37. Matsui H, Asou H, Inaba T. Cytokines direct the regulation of Bim mRNA stability by heat-shock cognate protein 70. Mol Cell. 2007;25(1):99-112.

38. Wilson MA, et al. The 1.1-A resolution crystal structure of $D J-1$, the protein mutated in autosomal recessive early onset Parkinson's disease. Proc Nat Acad Sci USA. 2003;100(16):9256-61.

39. Wang $B$, et al. Essential control of mitochondrial morphology and function by chaperone-mediated autophagy through degradation of PARK7. Autophagy. 2016;12(8):1215-28.

40. Ouseph MM, et al. Autophagy is induced upon platelet activation and is essential for hemostasis and thrombosis. Blood. 2015;126(10):1224-33.

41. Paul M, et al. Aggregation is impaired in starved platelets due to enhanced autophagy and cellular energy depletion. Platelets. 2019:30(4):487-97.

\section{Publisher's Note}

Springer Nature remains neutral with regard to jurisdictional claims in published maps and institutional affiliations.
Ready to submit your research? Choose BMC and benefit from:

- fast, convenient online submission

- thorough peer review by experienced researchers in your field

- rapid publication on acceptance

- support for research data, including large and complex data types

- gold Open Access which fosters wider collaboration and increased citations

- maximum visibility for your research: over $100 \mathrm{M}$ website views per year

At $\mathrm{BMC}$, research is always in progress.

Learn more biomedcentral.com/submissions 